\section{A novel non-invasive method to measure splenic filtration function in humans}

The detection of red cells with Howell-Jolly bodies and liver-spleen scintigraphy scanning are the currently used methods to assess splenic filtration function. The former is time-consuming, user-dependent and quantitative. The latter is semi-quantitative but uses radioactive material in an invasive, laborious manner. Here, we developed an automated, high-throughput, non-invasive and low-cost method to measure splenic filtration function accurately using flow cytometry.

Loss of splenic function results in short- and long-term potentially life-threatening complications including susceptibility to infections by encapsulated bacteria and thromboembolic events. ${ }^{1,2}$ Increasing evidence points to the need to preserve splenic function in various situations such as post-traumatic spleen injury, pancreatic neoplasia and hemolytic anemias. Adequate measurements of the residual function of the spleen are, therefore, crucial.
Howell-Jolly bodies (HJB) are $1 \mu \mathrm{m}$ DNA inclusion bodies that are the consequence of cytogenetic damage. In healthy individuals, HJB-containing red blood cells $(\mathrm{RBC})$ are found at very low frequency as $\mathrm{HJB}$ are cleared efficiently by the spleen. The spleen is a distinctive adapted lymphoid organ that serves as the largest filter of blood in the human body. The spleen comprises two critical structures: (i) the white pulp, containing the immune effector cells and (ii) the red pulp, composed of splenic sinuses and pulp cords that form a filtering structural entity. ${ }^{3}$ RBC flowing in the pulp cords are forced to deform and squeeze through narrow inter-endothelial slits in order to enter the venous circulation. During this process intracellular inclusions, such as HJB or parasites, are removed from the RBC, a phenomenon known as pitting. ${ }^{4,5}$ Identification of $\mathrm{HJB}$ in the circulation consequently serves as an indicator of altered splenic filtration function. ${ }^{6,7}$

To date, the gold standard for assessing splenic function remains liver-spleen scintigraphy scanning. This technique provides a qualitative and/or semi-quantitative measurement of splenic function by evaluating the
A

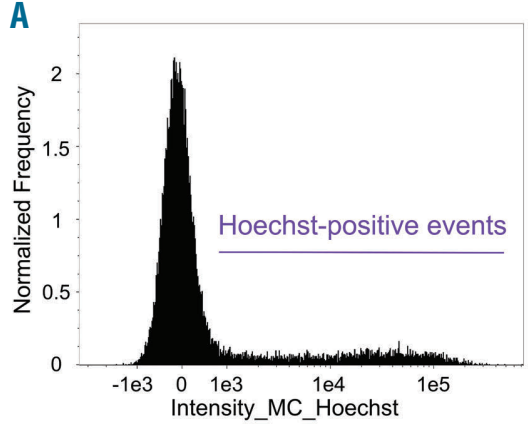

B
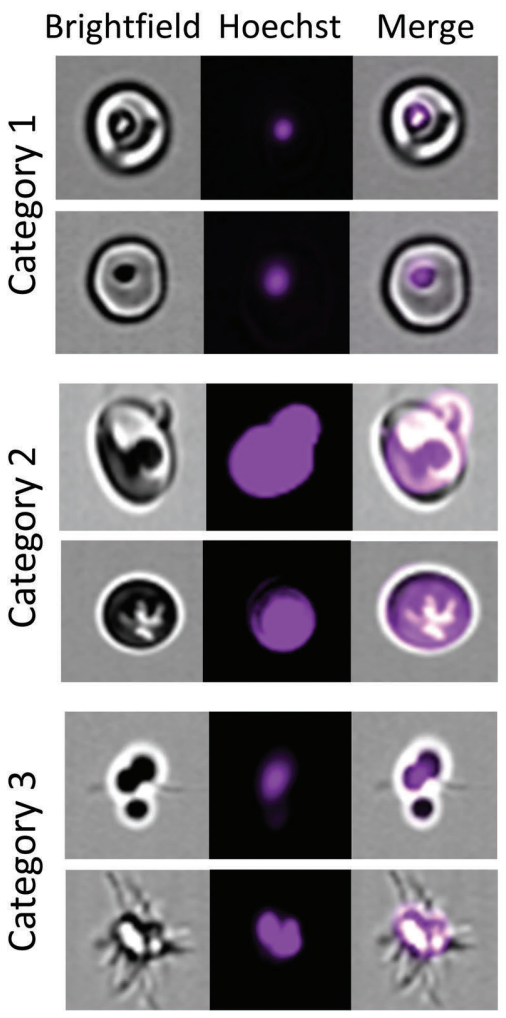

c

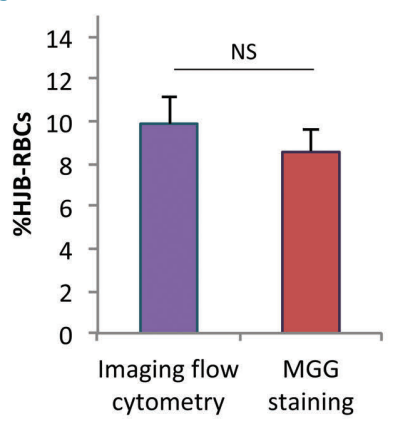

D

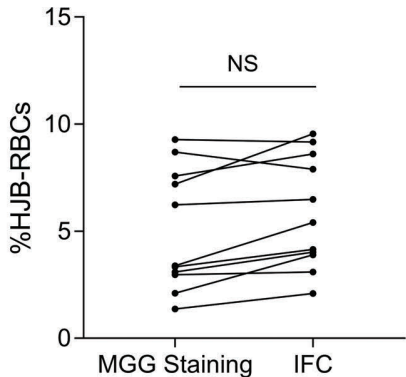

E

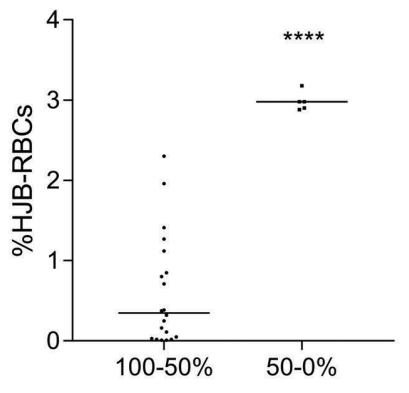

Figure 1. Detecting Howell-Jolly bodies using imaging flow cytometry. (A) Histogram representing the intensity of Hoechst staining in a splenectomized individual. (B) Images from imaging flow cytometry distinguishing three categories of cells: (i) RBC with HJB-like spots, (ii) RBC bound to Hoechst-positive particles and (iii) Hoechst-positive particles. (C, D) Graphs representing the percentage of HJB-RBC determined by the classical blood smear technique and imaging flow cytometry in (C) 10 splenectomized individuals (unpaired $t$ test, $P=0.07$ ) and (D) 11 adults with sickle cell disease (Wilcoxon test, $P=0.057$ ) (NS: non-significant). (E) Graphical representation of the percentage of HJB-RBC determined by imaging flow cytometry in 20 patients with a splenic uptake of $100-50 \%$ and seven patients with a splenic uptake of 50-0\% (MannWhitney test, $P<0.0001)$. MGG: MayGrunwald Giemsa, IFC: imaging flow cytometry. 
splenic uptake of either heat-denatured RBC or nanocolloids, labeled with technetium $-99 \mathrm{~m} .{ }^{8}$ Although these techniques have proven reliable, they are time-consuming, invasive and not readily available. The classically used method for assessing splenic dysfunction is, therefore, quantification of HJB-containing RBC by MayGrünwald Giemsa staining on blood smears. The percentage of $\mathrm{HJB}$-containing $\mathrm{RBC}$ is evaluated by counting 100-200 RBC under the microscope; the result is delivered as $\% \mathrm{HJB}$, a percentage higher than $1 \%$ reflecting loss of splenic function. This method is user-dependent and may be faulty because of the small number of counted cells. Another similar, commonly used method is to count "pitted" erythrocytes by interference contrast microscopy. 9 Spleen dysfunction does, however, occur gradually in many pathological conditions and is not accurately evaluated by a binary "filters/does not filter" state. A flow cytometry-based method using propidium iodide as a DNA dye to measure micronucleated reticulocytes was reported in $1996^{10}$ and later used in clinical studies to quantify HJB-containing RBC. ${ }^{11,12}$ As propidium iodide also stains RNA that is present in young RBC (reticulocytes), this method requires an efficient RNA digestion step to be accurate. In addition to issues regarding partial digestion, reticulocytes, which represent $0.5-1 \%$ of circulating $\mathrm{RBC}$ in healthy individuals, can reach high percentages in pathological situations such as hemolytic anemias, including sickle cell disease (SCD) and hereditary spherocytosis, which increases the risk of including significant numbers of false-positive events.

Here, we developed a simple non-invasive highthroughput technique based on flow cytometry to specifically detect $\mathrm{HJB}$-containing $\mathrm{RBC}$ and precisely quantify their percentage in the circulation. To do so, we used blood samples from splenectomized individuals, patients with SCD, and healthy donors. SCD is an autosomal recessive disease during which spleen injury occurs early in life due to vaso-occlusion caused by RBC sickling secondary to the polymerization of a mutated hemoglobin $(\mathrm{HbS}) .{ }^{13}$ The splenic dysfunction is associated with considerable morbidity and mortality ${ }^{14}$, including life-threatening acute splenic sequestration and an increased risk of infections. In SCD, the onset of spleen dysfunction occurs within the first years of life ${ }^{15}$ with the function decreasing thereafter to a state of complete loss, called auto-splenectomy.

In our method, we stained the HJB using Hoechst dye

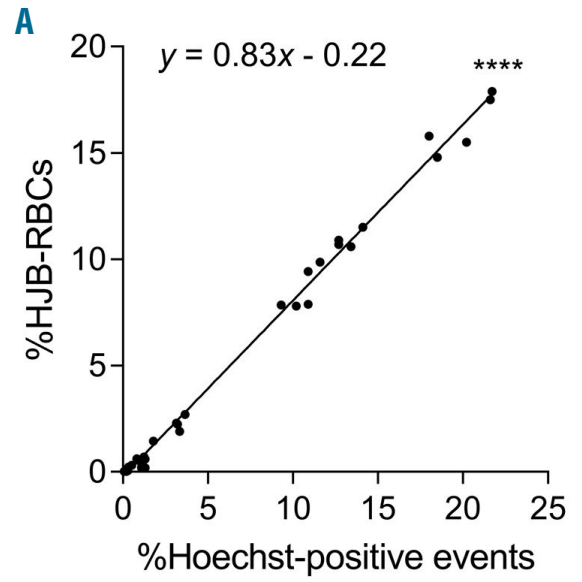

C

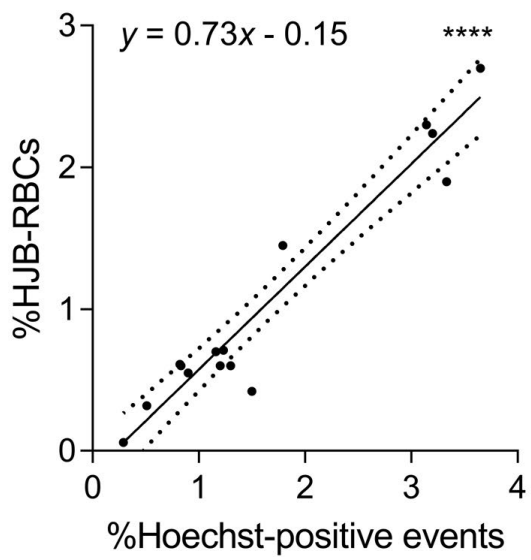

B

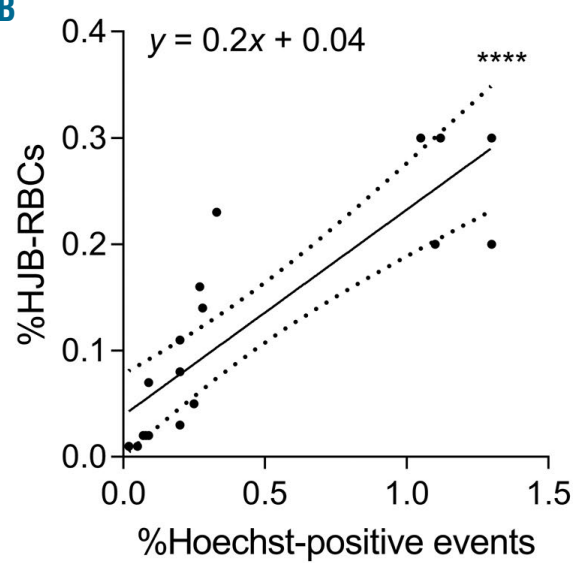

D

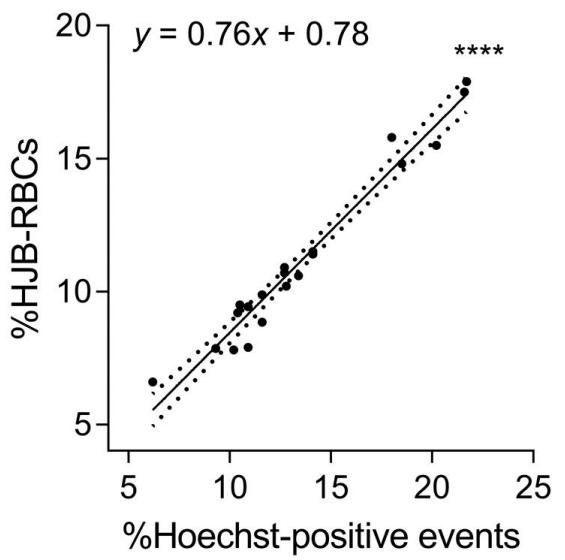

Figure 2. Relationship between \%Hoechst-positive events and \%HJB-RBC. (A) Linear regression relationship between \%Hoechst-positive events (x) and \%HJBRBC $(y)$ in 53 blood samples $(y=0.83 x-0.22)$. (B-D) Refinement of the linear regression according to three cut-offs of \%Hoechst-positive events: (B) <0.5\% $(n=18)(y=0.2 x+0.04),(C) 0.5-3.5 \%(n=14)(y=0.73 x-0.15)$, and (D) $>3.5 \%(n=21)(y=0.76 x+0.78)$. Correlation (Pearson correlation coefficient) and linear regression, $P<0.0001$. 
as this does not bind to RNA molecules. First, we used blood samples from splenectomized individuals to detect significant amounts of HJB-containing RBC (HJB-RBC). After removal of the buffy coat, the RBC were washed and incubated for $5 \mathrm{~min}$ in phosphate-buffered saline with Hoechst at $0.2 \%$. RBC were analyzed with a standard flow cytometer and percentages of Hoechst-positive events were determined (Online Supplementary Table S1, Online Supplementary Methods). As the results indicated high percentages of Hoechst-positive RBC, we repeated this experiment using seven blood samples from healthy donors. All samples showed percentages higher than 1\% (Online Supplementary Table S1) suggesting the presence of significant numbers of false-positive events. For further characterization, we used imaging flow cytometry and analyzed Hoechst-positive events in 50,000 RBC (Figure 1A,B). We could distinguish three categories: (i) RBC with HJB-like spots, (ii) RBC bound to Hoechst-positive particles and (iii) Hoechst-positive particles (Figure 1B). The Hoechst-positive particles were considered non-specific events, i.e. DNA released after lysis of neutrophils during the preparation steps, and were excluded using a combination of masks (Online Supplementary Figure S1A). This strategy markedly reduced the percentage of Hoechst-positive events in all blood samples (Online Supplementary Table S1) and all corresponding pictures thereafter showed RBC with HJB-like spots.

To validate this method, we compared the results with those obtained with classical May-Grünwald Giemsa staining in ten splenectomized individuals. HJB-RBC were counted manually within a total of 3,000 RBC (Online Supplementary Figure S1B). The results confirmed a robust correlation and therefore efficient gating and mask parameters to exclude false-positive events (Figure 1C). This was also the case when comparing both methods using blood samples from 11 adult patients with SCD (P1-P11) (Figure 1D).

We thereafter applied our imaging flow cytometry method to evaluate the percentage of HJB-RBC (\%HJB$\mathrm{RBC}$ ) in 25 very young SCD children (P12-P36; age range, 6-12 months) who underwent technetium-99m RBC splenic scintigraphy. Patients were divided into two groups according to their splenic uptake percentage: $(100 \%-50 \%)$ and (50\%-0\%). The two groups displayed marked differences, with the $(100 \%-50 \%)$ and $(50 \%-$ $0 \%$ ) groups showing medians of $0.35 \%$ and $2.98 \% \mathrm{HJB}-$ $\mathrm{RBC}$, respectively (Figure 1E).

Because imaging flow cytometry is not a readily available technology, we aimed at refining the standard flow cytometry method in order to allow accurate evaluation of \%HJB-RBC. We determined \%HJB-RBC by flow cytometry in the group of 53 individuals with various degrees of splenic function (36 SCD patients, 10 splenectomized individuals and 7 non-splenectomized healthy donors). The percentage of total Hoechst-positive events, determined by standard flow cytometry, was plotted against the percentage of HJB-containing RBC determined by imaging flow cytometry (Online Supplementary Table S1). We observed a robust linear regression between both parameters (Figure 2A), with the following equation: $y=0.83 x-0.22$, where $y$ is the $\% \mathrm{HJB}-\mathrm{RBC}$ and $x$ the \% of Hoechst-positive events generated by flow cytometry. We further refined this equation by dividing the 53 samples into three groups and generated three equations based on three cut-offs of Hoechst-positive events: $<0.5 \%, 0.5-3 \%$ and $>3 \%$ (Figure 2B-D).

Monitoring spleen function has been neglected because the methods used are labor-intensive, such as counting pitted or HJB-positive RBC, or invasive, such as liver-spleen scintigraphy scanning. Here, we describe an automated, non-invasive technique for the sensitive detection and evaluation of splenic filtration dysfunction. In contrast to the previously published flow cytometry technique, our method does not require cell fixation, RNA digestion steps or sample shipment. Our technique is simple and straightforward to implement in medical centers equipped with standard flow cytometers, thereby offering splenic function tests routinely. Such tests would be of great interest and might significantly affect the management and follow-up of patients with pathologies characterized by splenic injury, including SCD, thalassemia, red cell membrane disorders, celiac disease and cancer during chemotherapy. For instance, implementing our method in the early followup of children with SCD would play an important role in monitoring their infectious risk, a life-threatening consequence of this disease.

Sara El Hoss, ${ }^{1,2,3}$ Michaël Dussiot, ${ }^{4}$ Olivier Renaud, ${ }^{5,6,7,8}$ Valentine Brousse ${ }^{* 1,2,3,9}$ and Wassim El Nemer ${ }^{* 1,2,3,}$

'Biologie Intégrée du Globule Rouge UMR_S1134, Inserm, Univ. Paris Diderot, Sorbonne Paris Cité, Univ. de la Réunion, Univ. des Antilles; 'Institut National de la Transfusion Sanguine, Paris; ${ }^{3}$ Laboratoire d'Excellence GR-Ex, Paris; ${ }^{4}$ INSERM UMR1163, CNRS ERL8254, Laboratoire d'excellence GR-Ex, Université René-Descartes, Imagine Institute, Paris; Institut Curie, Paris Sciences et Lettres Research University, Paris; ${ }^{6}$ U934, Institut National de la Santé et de la Recherche Médicale, Paris;

${ }^{7}$ UMR3215, Centre National de la Recherche Scientifique, Paris; ${ }^{8}$ Cell and Tissue Imaging Facility

(PICT-IBiSA), Institut Curie, Paris and 'Service de Pédiatrie Générale, Hôpital Necker-Enfants Malades, Centre de Référence de la Drépanocytose, AP-HP, Paris, France

*VB and WEN contributed equally to this work.

Acknowledgments: we thank Dr Thierry Peyrard and Ms. Dominique Gien, Sirandou Tounkara and Eliane Véra at the Centre National de Référence pour les Groupes Sanguins for the management of blood samples, and Dr Arnaud Chene for his valuable help in performing MGG staining. This work was supported by the Institut National de la Santé et de la Recherche Médicale (Inserm), the Institut National de la Transfusion Sanguine and the Laboratory of Excellence GR-Ex, reference ANR-11-LABX-0051; $G R-E x$ is funded by the program "Investissements d'avenir" of the French National Research Agency, reference ANR-11-IDEX-000502. We acknowledge the PICT-IBiSA (Paris, France), member of the French National Research Infrastructure France-BioImaging (ANR-10-INBS-04), for microscopy experiments.

Funding: Sara El Hoss was funded by the Ministère de l'Enseignement Supérieur et de la Recherche (Ecole Doctorale BioSPC) and received financial support from the "Club du Globule Rouge et du Fer".

Correspondence: wassim.el-nemer@inserm.fr doi:10.3324/haematol.2018.188920

Information on authorship, contributions, and financial \& other disclosures was provided by the authors and is available with the online version of this article at www. haematologica.org.

\section{References}

1. Kristinsson SY, Gridley G, Hoover RN, Check D, Landgren O Long-term risks after splenectomy among 8,149 cancer-free American veterans: a cohort study with up to 27 years follow-up. Haematologica. 2014;99(2):392-398.

2. Lin JN, Lin CL, Lin MC, et al. Increased risk of hemorrhagic and ischemic strokes in patients with splenic injury and splenectomy: a nationwide cohort study. Medicine (Baltimore) 
2015;94(35):e1458.

3. Mebius RE, Kraal G. Structure and function of the spleen. Nat Rev Immunol. 2005;5(8):606-616.

4. Buffet PA, Safeukui I, Deplaine G, et al. The pathogenesis of Plasmodium falciparum malaria in humans: insights from splenic physiology. Blood. 2011;117(2):381-392.

5. Spencer RP, Pearson HA. The spleen as a hematological organ Semin Nucl Med. 1975;5(1):95-102.

6. Pearson HA, Spencer RP, Cornelius EA. Functional asplenia in sickle-cell anemia. N Engl J Med. 1969;281(17):923-926.

7. Brousse V, Buffet P, Rees D. The spleen and sickle cell disease: the sick(led) spleen. Br J Haematol. 2014;166(2):165-176.

8. Ehrlich CP, Papanicolaou N, Treves S, Hurwitz RA, Richards P. Splenic scintigraphy using Tc-99m-labeled heat-denatured red blood cells in pediatric patients: concise communication. J Nucl Med. 1982;23(3):209-213.

9. Casper JT, Koethe S, Rodey GE, Thatcher LG. A new method for studying splenic reticuloendothelial dysfunction in sickle cell disease patients and its clinical application: a brief report. Blood.
1976;47(2):183-188.

10. Dertinger SD, Torous DK, Tometsko KR. Simple and reliable enumeration of micronucleated reticulocytes with a single-laser flow cytometer. Mutat Res. 1996;371(3-4):283-292.

11. Harrod VL, Howard TA, Zimmerman SA, Dertinger SD, Ware RE. Quantitative analysis of Howell-Jolly bodies in children with sickle cell disease. Exp Hematol. 2007;35(2):179-183.

12. Rogers ZR, Wang WC, Luo Z, et al. Biomarkers of splenic function in infants with sickle cell anemia: baseline data from the BABY HUG Trial. Blood. 2011;117(9):2614-2617.

13. Pauling L, Itano HA, et al. Sickle cell anemia, a molecular disease. Science. 1949;109(2835):443.

14. Ware RE, de Montalembert M, Tshilolo L, Abboud MR. Sickle cell disease. Lancet. 2017;390(10091):311-323.

15. Pearson HA, Gallagher D, Chilcote R, et al. Developmental pattern of splenic dysfunction in sickle cell disorders. Pediatrics. 1985;76(3):392-397. 